\title{
Reduced immunogenicity of a third COVID-19 vaccination among recipients of allogeneic hematopoietic stem cell transplantation
}

Previous allogeneic hematopoietic stem cell transplantation (allo-HSCT) is a risk factor for severe COVID-19 with mortality rates that may exceed $20 \%{ }^{1,2}$ The efficiency of two doses of mRNA-based COVID-19 vaccines is reportedly lower in allo-transplanted patients than in healthy controls with rates of seronegativity or failure to seroconvert in $15-31 \% .^{3-6}$ In a study of allo-transplanted patients with insufficient responses to two doses of the BNT162b2 (Pfizer-BioBTech) mRNA vaccine, only $48 \%$ of patients reached a putative threshold (4,160 arbitrary units $[\mathrm{AU}] / \mathrm{mL}$, corresponding to 590 World Health Organization [WHO] standard binding antibody units $[\mathrm{BAU}] / \mathrm{mL}$ ) of immunoglobulin G (IgG) against the receptor-binding domain of the spike 1 (S1) protein (anti-RBD) following a third vaccine dose. ${ }^{7}$

Forty recipients of allo-HSCT for hematological malignancies were identified in local transplant registries of the Region Western Götaland (population of approximately 1.7 million) and accepted participation in this sub-study within the DurIRVac study (clinicaltrials gov. Identifier: Eu-
draCT no. 2021-000349-42) at the Sahlgrenska University Hospital. All participants gave written informed consent before enrolment. The DurIRVac study was approved by the Swedish Ethical Review Authority (permit no. 202003276, 2021-00374 and 2021-00539) and by the Swedish Medical Products Agency (permit no. 5.1-2021-11118). All patients fulfilled national criteria from the Public Health Agency of Sweden (www.folkhalsomyndigheten.se) for receiving a third dose namely: (i) having undergone transplantation within 3 years or (ii) having ongoing immunosuppressive treatment for graft-versus-host-disease (GvHD). The European Society for Blood and Marrow transplantation (EBMT) guidelines for COVID-19 vaccination were also followed (www.ebmt.org, version 6.0, May $31,2021)$. Three patients were excluded based on previously confirmed COVID-19. All enrolled patients $(n=37)$ had received two doses of COVID-19 mRNA vaccine $\geq 8$ weeks prior to screening.

The median time from transplantation to the third vaccination was 23 months (min-max 6-191). Twenty-one (57\%)

Table 1. Patient and treatment characteristics by responders and non-responders to the third dose of mRNA vaccine.

\begin{tabular}{|c|c|c|c|c|c|c|}
\hline & \multicolumn{3}{|c|}{ Anti-RBD IgG in serum } & \multicolumn{3}{|c|}{$\mathrm{T}$-cell reactivity (S1-Y) } \\
\hline & $\begin{array}{c}\text { Positive } \\
(\geq 14 \mathrm{BAU} / \mathrm{mL})\end{array}$ & $\begin{array}{c}\text { Negative } \\
(<14 \mathrm{BAU} / \mathrm{mL})\end{array}$ & $P$-value ${ }^{1}$ & $\begin{array}{c}\text { Positive } \\
(\geq 10 \mathrm{pg} / \mathrm{mL} \text { IFN-Y) }\end{array}$ & $\begin{array}{c}\text { Negative } \\
(<10 \mathrm{pg} / \mathrm{mL} \text { IFN-Y) }\end{array}$ & P-value ${ }^{2}$ \\
\hline All patients ${ }^{5}(n=37)$ & $31(84 \%)$ & $6(16 \%)$ & & $19(51 \%)$ & $18(48 \%)$ & \\
\hline Vaccine (Pfizer/Moderna) & $21 / 10$ & $3 / 3$ & & $13 / 6$ & $7 / 11$ & \\
\hline $\begin{array}{l}\text { Age in years at vaccination } \\
\text { median (range) }\end{array}$ & $60(19-78)$ & $63(32-72)$ & & $64(19-70)$ & $60(26-78)$ & \\
\hline $\begin{array}{l}\text { Median days between dose } 2 \text { and } \\
3 \text { (range) }\end{array}$ & $123(56-157)$ & $139(127-174)$ & $0.01^{3}$ & $123(74-149)$ & $127(56-174)$ & \\
\hline $\begin{array}{l}\text { Median months from allo-HSCT, } \\
\text { (range) }\end{array}$ & $26(6-188)$ & $19(13-191)$ & & $22(7-188)$ & $30(6-191)$ & \\
\hline Ongoing IST ${ }^{6}$ (yes/no) & $20 / 11$ & $5 / 1$ & & $9 / 10$ & $15 / 3$ & $0.046^{4}$ \\
\hline Ongoing prednisone (yes/no) & $16 / 15$ & $2 / 4$ & & $12 / 7$ & $13 / 5$ & $0.03^{4}$ \\
\hline \multicolumn{7}{|c|}{ 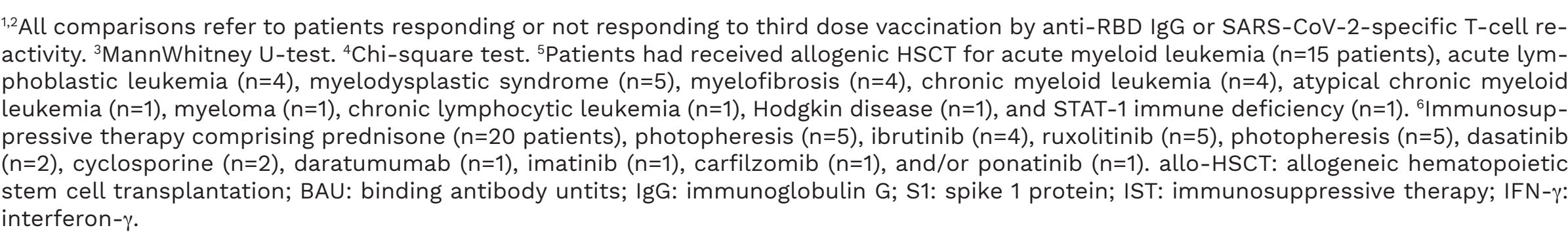 } \\
\hline
\end{tabular}


of participants had chronic GvHD and 25 (68\%) received immunosuppressive therapy (IST) at the time of vaccination (Table 1). Patients were given the same vaccine as in their initial two doses, i.e., either BNT162b2 (Pfizer-BioBTech Comirnaty ${ }^{\circledR} ; \mathrm{n}=24$ ) or mRNA-1273 (Moderna Spikevax $^{\circledR} ; n=13$ ) at a median 127 days (min-max 56-174) after the second dose. Peripheral blood was collected immediately before and 4 weeks (median 24 days, range, 19-30) after the third vaccination. Patients completed a questionnaire 2 weeks after the third dose to assess side-effects, categorized according to the CTCAE (common terminology criteria for adverse events) standards. Severity of GvHD was additionally assessed from medical records.

Chemiluminescent microparticle immunoassays were performed on serum using the automated Alinity system for analysis of IgG antibodies against RBD (SARS-CoV-2 IgG II Quant, Abbott, Illinois, USA) with levels reported in the WHO international standard $\mathrm{BAU} / \mathrm{mL}$ (quantitative detection range, 14-5,680 $\mathrm{BAU} / \mathrm{mL}$ ), which correlate well with neutralizing antibody levels. ${ }^{8}$ In order to assess T-cell responses $1 \mathrm{~mL}$ of peripheral blood, collected in heparinized tubes, was stimulated with peptides spanning the $\mathrm{N}$-terminal spike 1 (S1) domain of the SARS-CoV-2 surface glycoprotein. After 2 days of incubation at $37^{\circ} \mathrm{C}$ plasma was recovered for analysis of interferon- $\gamma$ (IFN- $\gamma$ ) by enzymelinked immunosorbant assay (ELISA). This assay captures SARS-CoV-2-specific reactivity of $\mathrm{CD}^{+}$and $\mathrm{CD} 8^{+} \mathrm{T}$ cells with high specificity and sensitivity. ${ }^{9} \mathrm{~S} 1$-induced IFN- $\gamma$ production is presented with levels in unstimulated samples subtracted using a limit of detection of $10 \mathrm{pg} / \mathrm{mL}$. Statistical analyses were performed using SPSS statistical software package (version 24) or GraphPad Prism software (version 9).

The majority (31/37, 84\%) of allo-HSCT patients responded to the third dose vaccination by increased anti-RBD IgG levels (Figure 1A). A subgroup (12/37, 32\%) achieved very high antibody levels $(>5,680 \mathrm{BAU} / \mathrm{mL})$. However, among the
14 patients seronegative prior to the third dose vaccination, six $(42 \%)$ remained seronegative 4 weeks after the third vaccine dose (Figure 1A). All patients who were seropositive before the third dose $(23 / 37,62 \%)$ achieved antibody responses exceeding $100 \mathrm{BAU} / \mathrm{mL}$, a level above which has been proposed to provide protection against COVID-19.10 The characteristics of responders and non-responders to the third vaccine dose are detailed in Table 1. No significant differences in serological responses were noted among patients with or without chronic GVHD or ongoing IST.

Regarding T-cell immune response, 18 of 37 (49\%) were devoid of measurable responses 4 weeks after the third vaccination (Figure 1B). T-cell responses tended to be lower in patients with chronic GVHD and were significantly diminished in patients receiving IST, in particular among those receiving prednisone (Table 1). Seronegativity prior to the third dose predicted poor humoral and cellular responses after vaccination. Treatment with IST was associated with insufficient $\mathrm{T}$-cell responses, more so than time from transplantation. Furthermore, four of five $(80 \%)$ of patients on ruxolitinib showed no T-cell reactivity. Of note, among the 14 patients who were seronegative for antiRBD IgG prior to the third dose, 11 (79\%) also lacked a Tcell response after three vaccine doses, compared with seven of $23(30 \%)$ among those seropositive prior to the third dose $(P<0.01$, chi-square test). Seronegativity prior to the third vaccination was non-significantly associated with ongoing GvHD (9/14 vs. 12/23 in seropositive patients) and IST (10/14 vs. 15/23). Additionally, a lower fraction of patients mounted SARS-CoV-2 specific T-cell responses than developed anti-RBD IgG after three vaccinations $(P<0.01$, chi-square test). Of six patients who remained seronegative after three vaccine doses, five (83\%) were also devoid of specific T-cells. Vaccine-reported adverse events were observed in $15(41 \%)$ patients after the third dose, with the majority of these categorized as mild local injection-related reactions. No exacerbations of GVHD were noted.
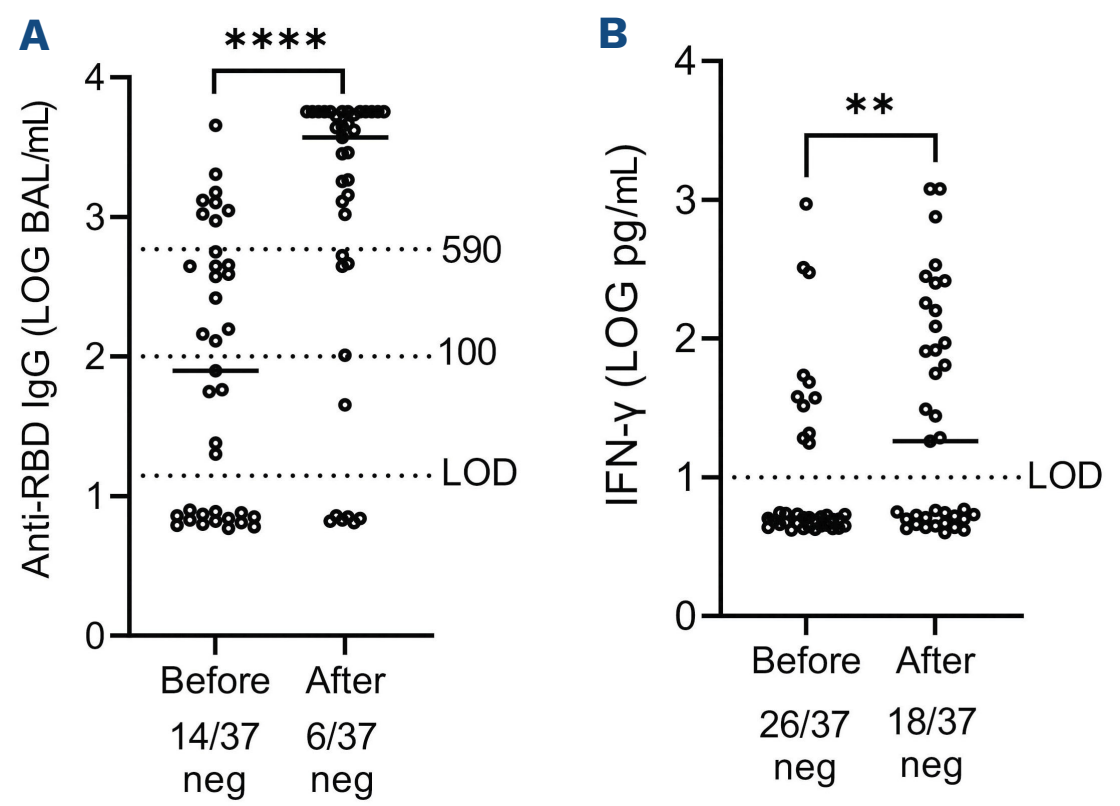

Figure 1. Serological and virus-specific T-cell responses to the spike 1 protein receptor region of SARS-CoV-2 before and after the third dose of of COVID-19 vaccines in allo-transplanted patients. (A) Shows serum levels of immunoglobulin G (IgG) against the receptor-binding domain (RBD). (B) Shows interferon- $\gamma$ (IFN$\gamma$ ) production in supernatant plasma following stimulation of whole blood with spike 1 peptides, reflecting reactivity of SARSCoV-2-specific $T$ cells. The upper dotted line represents the cutoff value of 590 binding antibody units [BAU]/mL (i.e., corresponding to 4,160 Abbott Arbitrary Units $[A U] / \mathrm{mL}$ ) while the middle dotted line corresponds to $100 \mathrm{BAU} / \mathrm{ml}$ and the lower dotted line represents the limit of detection (LOD) for respective assay. Statistical comparison by Wilcoxon matched pairs test $(\mathrm{n}=37)$. $P$-values are two-sided and are designated as follows: $\star * P<0.01, * * * * P<0.0001$. 
The main findings in this study were that a significant fraction of allo-transplanted patients failed to produce anti-RBD IgG (16\%) and that $48 \%$ of patients did not mount measurable SARS-CoV-specific $T$ cells despite three vaccinations. Our results confirm and extend a previous report of insufficient anti-RBD responses among allo-transplanted patients ${ }^{7}$ to imply that the inherent and treatment-induced T-cell deficiency associated with allotransplantation may translate into lack of COVID-19 mRNA vaccine efficacy. The interval between dose 2 and 3 was longer among patients remaining seronegative following the third dose, implying that a shorter interval between vaccinations may improve responses.

Our results additionally suggest that the SARS-CoV-2specific T-cell response to vaccination is more affected than the humoral response among allo-transplanted patients, based on the finding that a significantly higher fraction of patients showed complete deficiency of T-cell responsiveness to SARS-CoV-2-derived peptides compared with those remaining seronegative. Using the same T-cell assay, we have previously shown that 13 of 13 (100\%) of healthy donors developed detectable T-cell responses 4 weeks after the second SARS-CoV-2 vaccine dose. ${ }^{11}$ Notably, $35 \%$ of allo-transplanted patients lacked T-cell reactivity against $\mathrm{S} 1$ peptides despite mounting anti-RBD IgG. The clinical relevance of the observed T-cell deficiency remains to be established.

In conclusion, the third dose of COVID-19 mRNA vaccine resulted in elevated antibody titres and measurable SARSCoV-2-S1 T-cell responses in many allo-transplanted patients. However, a substantial proportion of patients did not respond by antibody formation and/or SARS-CoV-2-specific $T$ cells, highlighting the need for additional preventive measures and continued vigilance in this cohort.

\section{Authors}

Sigrun Einarsdottir, Anna Martner, ${ }^{2}$ Malin Nicklasson,, Hanna Grauers Wiktorin, ${ }^{2}$ Mohammad Arabpour, ${ }^{2,3}$ Andreas Törnell, ${ }^{2}$ Krista Vaht, ${ }^{1}$ Jesper Waldenström, ${ }^{3,4}$ Johan Ringlander, ${ }^{3,4}$ Tomas Bergström, Mats Brune, ${ }^{1}$ Kristoffer Hellstrand, ${ }^{2,3}$ Per Ljungman ${ }^{5}$ and Martin Lagging 3,4

1Department of Hematology and Coagulation, Institute of Medicine, Sahlgrenska Academy, University of Gothenburg, Gothenburg; ${ }^{2}$ TIMM Laboratory, Sahlgrenska Center for Cancer
Research, Department of Infectious Diseases, Institute of Biomedicine, Sahlgrenska Academy, University of Gothenburg, Gothenburg; ${ }^{3}$ Region Västra Götaland, Sahlgrenska University Hospital, Department of Clinical Microbiology, Gothenburg; ${ }^{4}$ Department of Infectious Diseases, Institute of Biomedicine, Sahlgrenska Academy, University of Gothenburg, Gothenburg and ${ }^{5}$ Department of Cellular Therapy and Allogeneic Stem Cell Transplantation, Karolinska Comprehensive Cancer Center, Karolinska University Hospital, Huddinge and Division of Hematology, Department of Medicine, Huddinge, Karolinska Institutet, Stockholm, Sweden.

Correspondence:

MARTIN LAGGING - martin.lagging@medfak.gu.se

https://doi.org/10.3324/haematol.2021.280494

Received: December 9, 2021.

Accepted: February 22, 2022.

Prepublished: March 3, 2022.

\section{Disclosures}

No conflicts of interest to disclose.

\section{Contributions}

$\mathrm{SE}$ and $\mathrm{ML}$ were responsible for designing and writing the protocol, conducting the study, extracting and analysing data, interpreting results, writing the letter, updating reference lists and creating the table and figure; $\mathrm{AM}$ and $\mathrm{KH}$ were responsible for designing and writing the protocol, extracting and analyzing data, interpreting results, writing the letter, updating reference lists and creating the table and figure; MN participated in interpreting results, writing the letter, and creating the figure; HGW, MA, and AT performed the Tcell assays as well as participated in extracting and analyzing data and interpreting results; KV, JW, and JR participated in extracting and analysing data and interpreting results; TB, MB, and PL participated in designing and writing the protocol, interpreting results, writing the letter, and updating the reference list.

\section{Funding}

This work was supported by the Swedish Medical Research Council (Vetenskapsrådet; grant number 2021-04779) and ALF Funds at Sahlgrenska University Hospital (grant number ALFGBG-438371).

\section{Data sharing statement}

The original data and protocols may be made available to other investigators after contact with the corresponding author.

\section{Clinical trial registration}

EudraCT 2021-000349-42

\section{References}

1. Sharma A, Bhatt NS, St Martin A, et al. Clinical characteristics and outcomes of COVID-19 in haematopoietic stem-cell transplantation recipients: an observational cohort study. Lancet Haematol. 2021;8(3):e185-e193. 


\section{LETTER TO THE EDITOR}

2. Ljungman $P$, de la Camara R, Mikulska M, et al. COVID-19 and stem cell transplantation; results from an EBMT and GETH multicenter prospective survey. Leukemia. 2021;35(10):28852894.

3. Redjoul R, Le Bouter A, Beckerich F, Fourati S, Maury S. Antibody response after second BNT162b2 dose in allogeneic HSCT recipients. Lancet. 2021;398(10297):298-299.

4. Bergman P, Blennow O, Hansson L, et al. Safety and efficacy of the mRNA BNT162b2 vaccine against SARS-CoV-2 in five groups of immunocompromised patients and healthy controls in a prospective open-label clinical trial. EBioMedicine. 2021;74:103705.

5. Dhakal B, Abedin S, Fenske T, et al. Response to SARS-CoV-2 vaccination in patients after hematopoietic cell transplantation and CAR T-cell therapy. Blood. 2021;138(14):1278-1281.

6. Ram R, Hagin D, Kikozashvilli N, et al. Safety and immunogenicity of the BNT162b2 mRNA COVID-19 vaccine in patients after allogeneic HCT or CD19-based CART therapy - a single-center prospective cohort study. Transplant Cell Ther. 2021;27(9):788-794.

7. Redjoul R, Le Bouter A, Parinet V, Fourati S, Maury S. Antibody response after third $B N T 162 b 2$ dose in recipients of allogeneic HSCT. Lancet Haematol. 2021;8(10):e681-e683.

8. Kristiansen PA, Page M, Bernasconi V, et al. WHO international standard for anti-SARS-CoV-2 immunoglobulin. Lancet. 2021;397(10282):1347-1348.

9. Brand I, Gilberg L, Bruger J, et al. Broad T cell targeting of structural proteins after SARS-CoV-2 infection: high throughput assessment of $\mathrm{T}$ cell reactivity using an automated interferon gamma release assay. Front Immunol. 2021;12:688436.

10. Hall VG, Ferreira VH, Ku T, et al. Randomized trial of a third dose of mRNA-1273 vaccine in transplant recipients. N Engl J Med. 2021;385(13):1244-1246.

11. Tornell A, Grauers Wiktorin H, Ringlander J, et al. Rapid cytokine release assays for analysis of SARS-CoV-2-specific T cells in whole blood. J Infect Dis. 2022 Jan 12 [Epub ahead of print]. 